gether in one volume a miscellaneous collection of such paragraphs, out of their natural contexts and having little in common but a particular range of temperatures.

In fact, however, some unity has been given to the present series by concentrating largely on one of the classic problems of this field: the behaviour of helium itself. In the present volume, as in its predecessor, almost half the space is devoted to the properties of helium at low temperatures in the solid, liquid and gaseous phases. Van Dijk and Durieux review the evidence leading to the Leyden 1955 vapour-pressure scale, and also discuss briefly the American 1955 scale. It is lamentable that there should be at present two 'rival' temperature scales, and unfortunate that Keller's criticisms of the Leyden scale appeared too recently to be considered in this review. Other articles discuss the properties of liquid helium in slits, in films and below $1^{\circ} \mathrm{K}$., and of solid helium. Lastly, there is a lengthy discussion by de Boer of 'solid-like' models of liquid helium, which to some extent complements the more realistic but less rigorous approach described by Feynman in the previous volume.

In contrast to this liberal treatment of helium, the other classic problem of low-temperature physics, superconductivity, is represented here by only one short article on superconducting alloys and compounds. Indeed, in the two volumes together, five times as many pages are devoted to the properties of helium as to superconductivity. This presumably reflects editorial policy : it need scarcely be said that it in no way reflects the relative amount of progress to be reported in the two fields.

The remaining seven articles cover a miscellany of topics, including conduction in metals, properties of semiconductors, the de Haas-van Alphen effect, paramagnetic relaxation, the rare earths, nuclear orientation, and lattice specific heats, and moreover they differ rather widely in level of presentation. In view of this, it is difficult to recommend the volume unreservedly to a general audience. Those working specifically on the properties of helium, however, will no doubt find it a useful companion to the previous volume.

R. G. Chambers

\section{PERCEPTION AND UTILIZATION OF LIGHT}

Radiation Biology

Edited by Alexander Hollaender, with the co-operation of Sterling B. Hendricks. Vol. 3: Visible and Near-Visible Light. (Prepared under the Auspices of the Committee on Radiation Biology, Division of Biology and Agriculture, National Research Council -National Academy of Sciences, Washington, D.C.) Pp. viii + 765. (London : MeGraw-Hill Publishing Company, Ltd., 1956.) $75 s$.

$7 \mathrm{HE}$ subject of this volume is of special significance because the transformations of energy with many essential components of living organisms are 'tuned' to radiation in the visible and near visible spectrum. The variety of problems under investigation which belong to photobiology is very large indeod, and it might seem too much to expect that all aspects could be contained in a single volume. Yet, as may be seen from what follows here, the sub. ject is covered in the fifteen chapters remarkably well.
'The first three chapters deal with physical problems; first a discussion of energy exchanges involving atoms and molecules, where the use of potential diagrams is developed in relation to the theory of absolute reaction rates. This is followed by a most valuable exposition of the physics of light absorption by molecules ; two important classes of biological pigments, carotenoid (polyene) and tetrapyrrolic pigments (chlorin and porphin), are dealt with. This chapter gives an account of some dramatic achievements in this field. 'Thirdly, there is a longer section on the practical physics of the radiant energy, which is most ably presented. The next four sections deal with photosynthesis : the quantum requirement, the mechanism of photosynthesis, the spectroscopy of the pigments and the formation of chlorophyll in the plant. The discussion on the mechanism of photosynthesis where the physical, physiological, and biochemical approaches are combined is well documented and of a distinctly individual character. The part played by light in the nitrogen cycle of plant growth is discussed separately. I think the climax is reached in Chapters 9 and 10, which give with masterly authority accounts of phototropism and photoperiodism. The writing is well sustained in the following two chapters on seed germination and the relation of light to the physiology of the cell. The next two chapters are concerned with eyes. Although the account of electrical phenomena in vision contains a wealth of information, I was sorry not to find more about human colour vision, more about the biochemistry of visual pigments and just a little about quantum requirements, for aspects analogous to these have whole sections devoted to them in photosynthesis. The account of invertebrate photoreceptors is of a quality which whets the appetite for more (actually, we are promised this by the contributors next year); this chapter, together with the second, on excitation of polylenes and porphyrins, are perhaps largely responsible for the unique character of the book. The last contribution, on the old-established subject somewhat strangely known as 'photodynamic action', contains recent material which is fundamental both to photobiology and to radiobinlogy as a whole. On the use of the various symbols for defining absorption and extinction coefficients, information is scanty and it is not collected together in any one section. We are given good descriptions of the natural pigments and their absorption spectra but the reader may find difficulties with the quantitative data. The usual convention has been to employ common logarithms in extinction coefficients and natural logarithms in absorption coefficients. The curves given for the chlorophylls have the ordinates labelled absorption but the values are in common logarithms: thus the reader may be compelled to consult other literature unnecessarily. I would have welcomed some selected tables of extinction coefficients for several of the pigments and feel tempted to appeal to future editors concerned with spectroscopic data to allow the publication of data in tabular form rather than, or in addition to, the curves from which it is both difficult and tiresome to get values for different wave. lengths.

The editor and his distinguished colleagues are to be congratulated on their skill in producing this volume, which is a valuable addition to literature on general biology. It should appeal to a wide range of advanced students. Though the contributions were mainly completed in 1951 they are an indispensable guide to current research.
Robert HILL 